Gynäkologe 2019·52:366-368 https://doi.org/10.1007/s00129-019-4406-x Online publiziert: 27. Februar 2019 ๑ Springer Medizin Verlag GmbH, ein Teil von Springer Nature 2019

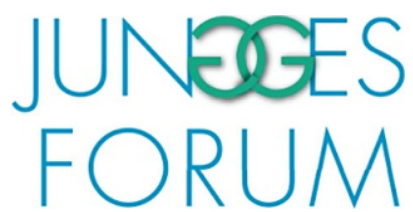

CrossMark

\author{
Martin Weiss ${ }^{1,3} \cdot$ Fabian Winterholler $^{2,3}$ \\ 'Department für Frauengesundheit, Tübingen, Deutschland \\ ${ }^{2}$ Klinik für Frauenheilkunde und Geburtshilfe, Universitätsklinik der Paracelsus Medizinischen \\ Privatuniversität, Klinikum Nürnberg, Nürnberg, Deutschland \\ ${ }^{3}$ Repräsentanz der DGGG und Fachgesellschaften, Deutsche Gesellschaft für Gynäkologie und \\ Geburtshilfe e. V., Berlin, Deutschland
}

\title{
Das Junge Forum der Deutschen Gesellschaft für Gynäkologie und Geburtshilfe stellt sich vor
}

\section{Für die Verbesserung der Facharztweiterbildung für Gynäkologie und Geburtshilfe}

Vertretung der Interessen des JF aus und sind jeweils ein stimmberechtigtes Mitglied des Vorstandes. Regelmäßig stattfindende Treffen des Arbeitskreises und der Sprecher rücken die Diskussion und die Strategieentwicklung zu tagesaktuellen und zukünftigen Herausforderungen des Fachgebietes und der Weiterbildung zum Facharzt für Gynäkologie und $\mathrm{Ge}$ burtshilfe in den Fokus unserer Arbeit. Um ein möglichst breites Spektrum an Interessen vertreten zu können, arbeitet das JF eng mit den Vertreterinnen und Vertretern der regionalen Gesellschaften, dem Berufsverband und den Jungen Foren angrenzender Fachgesellschaften sowie des europäischen Auslands zusammen.

\section{Perspektive Frauenärztin/ Frauenarzt}

Von der Pränatalphase bis zum Tod - ohne Zweifel ist das Fachgebiet Gynäkologie und Geburtshilfe eines der umfang- und facettenreichsten medizinischen Disziplinen. Neben dem Facharzt für Gynäkologie und Geburtshilfe sind Zusatzweiterbildungen im Bereich Gynäkologische Endokrinologie und Reproduktionsmedizin, Gynäkologische Onkologie sowie Spezielle Geburtshilfe und Perinatalmedizin möglich.
Außerdem sind die Bereiche Pränatalmedizin, Geburtshilfe, Prävention, Familienplanung, Endokrinologie und Reproduktionsmedizin, Gynäkologie und Urogynäkologie, konservative und operative Onkologie nur einige Beispiele, um die Vielfalt des Faches aufzuzeigen. Der Spagat zwischen sensibler, ganzheitlicher und generationenübergreifender Versorgung unterschiedlichster Organsysteme der Frau einerseits, wissenschaftlicher Exzellenz und rasanter Entwicklung anspruchsvoller, klinischer Hightech Verfahren andererseits stellt besondere Herausforderungen an die Facharztweiterbildung für Gynäkologie und Geburtshilfe. Gleichsam sind der Abwechslungsreichtum und die Fülle an beruflichen Perspektiven ein besonders reizvoller Aspekt unseres Fachgebietes. Die „Frauenheilkunde und Geburtshilfe“ bietet viele verschiedene Tätigkeitsfelder, die nach individuellen Vorlieben und Berufsvorstellungen in Voll- oder Teilzeit, ambulant oder stationär ausgeübt werden können. Durch die Praxisniederlassung oder die Anstellung als niedergelassene Frauenärztin oder niedergelassener Frauenarzt ist eine nicht-operative Tätigkeit und die ärztliche und präventive Betreuung von Frauen und ganzen Familien teils über Generationen hinweg möglich. Wir im JF haben es uns zum 
Ziel gemacht, bereits Medizinstudierenden unser Fachgebiet praktisch näher zu bringen und sie für die Gynäkologie und Geburtshilfe zu begeistern. Gleichwohl begleiten wir alle unsere Kolleginnen und Kollegen auf Ihrem Weg durch die Facharztweiterbildung - in allen Phasen. Das Engagement lohnt sich - mit rund 18.500 Fachärztinnen und Fachärzten [1] ist die Frauenheilkunde unter den personell stärksten und medizinpolitisch einflussreichsten Fachgebieten in Deutschland vertreten.

\section{Unsere Ziele}

\section{Verbesserung der Weiterbildung}

Wir setzen uns für unsere Kolleginnen und Kollegen in den Kliniken und für eine flächendeckend hochwertige Facharztweiterbildung ein. Laut Muster-Weiterbildungsordnung (M-WBO) der Bundesärztekammer dauert diese mindestens 60 Monate. Das entspricht 5 Jahren Weiterbildung, die sowohl in den Bereichen der einzelnen gynäkologischen und geburtshilflichen Schwerpunktweiterbildungen, im ambulanten Bereich wie auch in begrenztem Umfang in einem anderen medizinischen Gebiet absolviert werden können. Wir möchten auf gegebenenfalls bestehende Schwächen im System aufmerksam machen und diese bekämpfen. Die Themen „Vereinbarkeit von Familie und Beruf" ebenso wie "gleichwertige Karrierechancen für Ärztinnen und Ärzte mit Kind" sind in kaum einem anderen Fachgebiet so aktuell, wie sie es in der Gynäkologie und Geburtshilfe sind. Immerhin haben wir es bei den Mitgliedern der DGGG unter 35 Jahren aktuell mit einem Anteil an Ärztinnen von knapp 89\% zu tun. Eine deutschlandweit durch das JF durchgeführte Umfrage mit 437 Teilnehmerinnen und Teilnehmern zeigte auch für unser Fachgebiet klar identifizierbare Defizite auf [2].

\section{Umfangreiche Kurs- und Fortbildungsangebote für Assistenzärztinnen und Assistenzärzte}

Eine wesentliche Aktivität des JF ist die Konzeption und Durchführung von
Basiskursen u.a. in den Bereichen Geburtshilfe, minimalinvasive Chirurgie (MIC), Endokrinologie, Urogynäkologie, Psychosomatische Grundversorgung und Notfallmanagement. In Kooperation mit der Arbeitsgemeinschaft Gynäkologische Onkologie (AGO) der DGGG und der deutschen Krebsgesellschaft befasst sich das JF intensiv mit der Förderung des Nachwuchses in der Aus- und Weiterbildung sowie in Wissenschaft und Forschung im Bereich der gynäkologischen Onkologie. Ein eigens eingerichteter Basiskurs „Gynäkologische Onkologie“ vermittelt Grundkenntnisse zum Verständnis, Diagnostik und Therapie der relevanten Tumoren der weiblichen Geschlechtsorgane, einschließlich der Tumoren der Brust.

\section{Nachwuchsförderung}

Gemeinsam mit der Kommission Nachwuchsförderung der DGGG hat sich das JF die Gewinnung und die Begeisterung des ärztlichen Nachwuchses für das Fach Gynäkologie und Geburtshilfe auf die Fahnen geschrieben. Eine eigens initiierte Imagekampagne wird ab dem kommenden Jahr auf das Fachgebiet aufmerksam machen - jung, frech und mit einem Augenzwinkern. „Wir sind ein modernes Fachgebiet und haben dem medizinischen Nachwuchs einiges zu bieten", so der Präsident der DGGG Prof. Dr. Anton Scharl. „Wir wollen unsere zukünftigen Kolleginnen und Kollegen auf uns aufmerksam machen. Die Imagekampagne und die Arbeit des Jungen Forums sind die Schlüssel hierfür." Neben Stipendien und kostenlosen Zugängen zu Fachkongressen der DGGG und deren Arbeitsgemeinschaften, veranstaltet das JF jährlich eine zweitägige „Summer School“ für Studentinnen und Studenten. „2019 werden wir am Department für Frauengesundheit in Tübingen sein. Wir haben ein großartiges fachliches und kulturelles Programm auf die Beine gestellt, das die jungen Teilnehmerinnen und Teilnehmer begeistern wird“, so Dr. Martin Weiss, der gemeinsam mit Dr. Vera Hepp das Amt der Sprecherin und des Sprechers des JF der DGGG im November 2018 übernommen hat.
Internationale Zusammenarbeit Weiterbildung und Mobilität verbessern über die Bundesgrenzen hinaus

Das JF der DGGG pflegt eine mehrjährige aktive Kooperation mit dem European Network of Trainees in Obstetrics and Gynecology (ENTOG). Im Fokus der Zusammenarbeit steht die Förderung der ärztlichen Mobilität und die Pflege der freundschaftlichen Beziehungen innerhalb Europas durch regelmäßigen Austausch und klinische Hospitationen von Assistenzärztinnen und Assistenzärzten in Europäischen Nachbarländern sowie durch die aktive Teilnahme an Kongressen des European Board and College of Obstetrics and Gynecology (EBCOG). Zukünftig wird die Stärkung der Wahrnehmung für das im letzten Jahr erschienene EBCOG Paneuropean Curriculum for Training (EBCOG-PACT) und dessen Evaluation für die Facharztweiterbildung in Deutschland eine wichtige Rolle einnehmen. Mit EBCOG-PACT wird didaktisch und teilweise auch inhaltlich ein einheitlicher europäischer Standard für die Weiterbildung zum Facharzt für Gynäkologie und Geburtshilfe geschaffen und damit die Mobilität des medizinischen Personals sowie der Transfer von Wissen und Fertigkeiten innerhalb Europas gefördert. EBCOGPACT ist das Resultat einer Förderung durch das ERASMUS+ Programm, das von der Europäischen Union finanziert wurde. Die Implementierung in Deutschland ist fraglich - das JF der DGGG wird sich für die Interessen der Kolleginnen und Kollegen einsetzen.

Es grüßen Sie herzlich im Namen des JF der DGGG

Martin Weiss und Fabian Winterholler

Machen Sie sich selbst ein Bild von der vielseitigen Arbeit des Jungen Forums der Deutschen Gesellschaft für Gynäkologie und Geburtshilfe! Besuchen Sie uns unter:

- https://www.dggg.de/weiterbildungdggg-nachwuchs/junges-forum/ 
- https://www.facebook.com/ - junges forum der dggg e.v.

Bei Fragen stehen wir gerne unter jungesforum@dggg.de zur Verfügung.

\section{Korrespondenzadresse}

Dr. med. Martin Weiss
Repräsentanz der DGGG und
Fachgesellschaften, Deutsche
Gesellschaft für Gynäkologie
und Geburtshilfe e.V.
Hausvogteiplatz 12,
10117 Berlin, Deutschland
junges-forum@dggg.de

\section{Literatur}

1. https://www.bundesaerztekammer.de/ fileadmin/user_upload/downloads/pdf-Ordner/ Statistik2017/Stat17AbbTab.pdf. Zugegriffen: 01.12 .2018

2. Neimann J, Knabl J, Puppe J, Bayer CM, Gass P, Gabriel L, Seelbach-Goebel B, Lermann J, Schott S (2017) Duty Rosters and Workloads of Obstetricians in Germany: Results of a Germanywide Survey. Geburtshilfe Frauenheilkd 77:894

\section{NIPT nur nach besonderer Indikationsstellung einsetzen}

Der Gemeinsame Bundesausschuss (G-BA) diskutiert derzeit darüber ob ein Bluttest auf kindliche Chromosomenanomalien in den Leistungskatalog der gesetzlichen Krankenkassen aufgenommen werden soll. Damit müsste er nicht mehr ausschließlich als individuelle Gesundheitsleistung (IGeL) von den Schwangeren selbst bezahlt werden.

In einem Positionspapier fordert der BVNP (Berufsverband niedergelassener Pränatalmediziner) klare Rahmenbedingungen für diesen Fall und warnt gleichzeitig auch davor, ihn unkritisch einzusetzen. Der Test ersetze nicht die bisherige Diagnostik und müsse von einem klar definierten Katalog qualitätssichernder Maßnahmen begleitet werden, heißt es dort unter anderem.



(c) EduardHarkonen / Getty Images / iStock

Das besondere an der als NIPT (nichtinvasiver pränataler Test) bezeichneten Methode ist, dass die für die Untersuchung notwendige kindliche DNA aus Zellen gewonnen wird, die im Blut der Mutter zirkulieren. Auf einen invasiven Eingriff in den Uterus - wie er etwa bei der Amniozentese oder der Chorionzottenbiopsie nötig ist - kann verzichtet werden. „Ein Vorteil des NIPT bietet sich jedoch nur im Falle eines negativen Testergebnisses, denn dieses schließt mit hoher Wahrscheinlichkeit eine Trisomie 21 aus," sagt Professor Dr. med. Alexander Scharf, Präsident des BVNP. Umgekehrt reiche der NIPT aber nicht aus, um eine Trisomie 21 sicher zu diagnostizieren. Ein auffälliges Ergebnis müsse daher immer in einer anschließenden invasiven Untersuchung überprüft werden. Hierüber müssten die Schwangeren ebenso aufgeklärt werden, wie über die Tatsache, dass der NIPT nicht alle Chromosomenstörungen adressiert und keinerlei Aussagen über die wesentlich häufigeren, nicht genetisch bedingten körperlichen Fehlbildungen des Ungeborenen erlaube. Diese seien nur im Ultraschall zu erkennen. „Der NIPT sollte daher nur im Zusammenhang mit einer Ultraschalluntersuchung unter Einschluss der Beurteilung der fetalen Nackentransparenz vorgenommen werden", sagt Professor Scharf - und zwar von Ärzten, die beide Untersuchungen aus einer Hand anbieten könnten.

Bei einem vom BVNP favorisierten und vorgeschlagenen Modell würde der NIPT nicht flächendeckend angeboten, sondern könnte lediglich nach besonderer Indikationsstellung als GKV-Leistung abgerechnet werden. Damit sei auch sichergestellt, dass das Angebot eines NIPT eng in die bisherigen Strukturen der Pränataldiagnostik eingebunden werde. "Die vereinfachende Werbung suggeriert schnelle Gewissheit und blendet die Folgen eines positiven Testergebnisses weitgehend aus", gibt Professor Scharf zu Bedenken. Weil die Wirklichkeit nicht so einfach sei, müsse den Schwangeren eine qualifizierte medizinische und psychosoziale Beratung zur Verfügung stehen. Auch müsse gewährleistet sein, dass die Betroffenen auf Anschlussuntersuchungen, die unter Umständen notwendig werden, nicht lange warten müssten. Dazu zählen auch die invasiven Techniken der Nabelschnurpunktion, Chorionzottenbiopsie oder Amniozentese.

Nicht zuletzt hält der BVNP das Angebot eines flächendeckenden NIPT ohne Indikation auch aus ethischen Gründen für fragwürdig. Der Verband begrüßt daher eine parlamentarische Initiative, mit der Bundestagsabgeordnete mehrerer Fraktionen eine Debatte zum NIPT und anderen pränatalen Tests im Bundestag anstoßen wollen. In einem hierzu vorgelegten Positionspapier fordern die Abgeordneten, die Einführung solcher Tests in Zukunft nicht ohne eine parlamentarische und gesellschaftliche Debatte vorzunehmen. Hierbei sollten auch die Positionen behinderter Menschen und ihrer Angehörigen stärker berücksichtigt werden.

Quelle: Berufsverband niedergelassener Pränatalmediziner (BVNP), www.bvnp.de 